\title{
Caracterização das raízes do polinômio do terceiro grau e alguns resultados
}

Characterization of the roots of the third degree polynomial and some results

\section{LUÍS YAMAOKA ${ }^{\mathrm{a}}$}

\begin{abstract}
Resumo
Neste artigo apresentamos um procedimento para caracterizar as raízes do polinômio do $3^{\mathrm{o}}$ grau $f \in \mathbb{R}[x]$ recorrendo a fatos do Cálculo. Ademais, impondo condições aos coeficientes de $f \in \mathbb{R}[x]$, estabelecemos uma comparação entre a parte real de suas raízes complexas não reais conjugadas e o(s) ponto(s) crítico(s) de $\mathbf{f}: \mathbb{R} \rightarrow \mathbb{R}$.
\end{abstract}

Palavras-chave: Polinômio do terceiro grau, Caracterização das raízes, Parte real.

\begin{abstract}
In this paper we present a procedure to characterize the roots of the 3rd degree polynomial $f \in \mathbb{R}[x]$ using facts of Calculus. Furthermore, by imposing conditions on the coefficients of $f \in \mathbb{R}[x]$, we establish a comparison between the real part of their conjugated non-real complex roots and the critical point(s) of $\mathbf{f}: \mathbb{R} \rightarrow \mathbb{R}$.
\end{abstract}

Keywords: Third degree polynomial, Root characterization, Real part.

MSC2010: 12D10

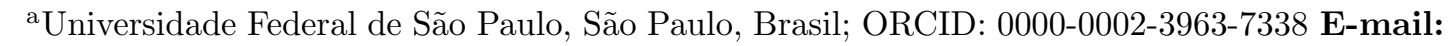
luis.yamaoka@unifesp.br 


\section{Introdução}

Denotamos por $\mathbb{C}[x]$ o conjunto dos polinômios na indeterminada $x$ com coeficientes em $\mathbb{C}$. Sejam $f \in \mathbb{C}[x]$ e $r \in \mathbb{C}$. Dizemos que $r$ é raiz de $f$ se o número complexo $f(r)$ é igual a zero. Consideremos $\mathbb{R}[x]$ o conjunto dos polinômios na indeterminada $x$ com coeficientes em $\mathbb{R}$. A partir daqui, abordaremos os polinômios do $3^{\underline{0}}$ grau em $\mathbb{R}[x]$.

É fato conhecido que cada polinômio do $3^{0}$ grau $f \in \mathbb{R}[x]$ admite uma dentre as seguintes possibilidades para as suas raízes (veja, por exemplo, [7]):

- três raízes reais distintas;

- uma raiz real de multiplicidade 1 e outra raiz real distinta de multiplicidade 2 ;

- uma raiz real de multiplicidade 3 ;

- uma raiz real e duas raízes complexas não reais conjugadas.

Cada uma dentre as quatro possibilidades supracitadas é uma caracterização das raízes de um polinômio do $3^{0}$ grau $f \in \mathbb{R}[x]$. Na Seção 2 daremos os detalhes a respeito de cada caracterização. O intuito deste artigo é valer-se do Cálculo para caracterizar as raízes de $f \in \mathbb{R}[x]$ e, sob condições impostas aos seus coeficientes, realizar uma comparação entre a parte real das suas raízes complexas não reais e o(s) ponto(s) crítico(s) de $\mathbf{f}: \mathbb{R} \rightarrow \mathbb{R}$, em que $\mathbf{f}$ é a função polinomial do $3^{0}$ grau definida por $f$.

As ferramentas empregadas para alcançar os resultados aqui apresentados podem ser obtidas em um texto de Cálculo e na coleção Fundamentos de Matemática Elementar. Por exemplo, os conjuntos numéricos, as funções quadráticas, a definição de função sobrejetora e equações e inequações irracionais estão expostos em [4]. Números complexos, polinômios e equações polinomiais são tratados em [3]. Posições de um ponto em relação ao sistema, em [2]. As derivadas são contempladas em [1] e em [5]. Temos como objetivo demonstrar o proposto neste trabalho sem recorrer à fórmula da solução geral da equação cúbica. Tais raízes podem ser obtidas por um software matemático. As formas dos gráficos das funções polinomiais do $3^{\underline{0}}$ grau exibidas na Seção 2 também podem ser encontradas em [6]. 


\section{A caracterização das raízes}

Seja $\mathbf{f}: \mathbb{R} \rightarrow \mathbb{R}$ dada por $\mathbf{f}(x)=a x^{3}+b x^{2}+c x+d, a, b, c, d \in \mathbb{R}, a>0, \forall x \in \mathbb{R}$. Definimos a vizinhança de um ponto $x_{0}$ pertencente ao domínio de $\mathbf{f}, D(\mathbf{f})=\mathbb{R}$, um intervalo $V=] x_{0}-\delta, x_{0}+\delta[$, onde $\delta$ é um número real positivo. Consideremos a derivada de $\mathbf{f}$ :

$$
\mathbf{f}^{\prime}(x)=3 a x^{2}+2 b x+c .
$$

Definimos um ponto crítico de $\mathbf{f}$ como sendo o valor de $x \in \mathbb{R}$ para o qual $\mathbf{f}^{\prime}(x)=0$. A forma do gráfico de $\mathbf{f}$ depende do número de pontos críticos de $\mathbf{f}$. Deste modo, calculamos o discriminante de $\mathbf{f}^{\prime}$ :

$$
\Delta=4 b^{2}-12 a c .
$$

Se $\Delta>0$, $\mathbf{f}$ possui 2 pontos críticos; se $\Delta=0$, $\mathbf{f}$ possui 1 ponto crítico; se $\Delta<0, \mathbf{f}$ não possui ponto crítico. Logo, temos as 3 possíveis formas para o gráfico de $\mathbf{f}$ :

1. $1^{\text {a }}$ forma $(\Delta>0)$

Como $a>0$, os pontos críticos $p_{M}$ e $p_{m}$ são tais que

$$
p_{M}=\frac{-2 b-\sqrt{4 b^{2}-12 a c}}{6 a}<p_{m}=\frac{-2 b+\sqrt{4 b^{2}-12 a c}}{6 a} .
$$

Analisando a variação do sinal de $\mathbf{f}^{\prime}$, temos:

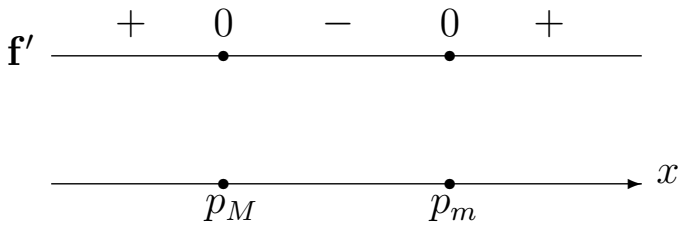

Como $\mathbf{f}^{\prime}(x)>0$ para $\left.x \in\right]-\infty, p_{M}[$ e para $x \in] p_{m}, \infty[$, segue que f é estritamente crescente em $]-\infty, p_{M}[$ e em $] p_{m}, \infty\left[\right.$; $\operatorname{como~}^{\prime}(x)<0$ para $\left.x \in\right] p_{M}, p_{m}[$, segue que $\mathbf{f}$ é estritamente decrescente em $] p_{M}, p_{m}\left[\right.$. Ademais, como $\mathbf{f}^{\prime}$ é positiva à esquerda e negativa à direita de $p_{M}$, decorre que $p_{M}$ é ponto de máximo local de $\mathbf{f}$, isto é, existe vizinhança $V$ de $p_{M}$ tal que $\mathbf{f}(x) \leq \mathbf{f}\left(p_{M}\right), \forall x \in V$. Como $\mathbf{f}^{\prime}$ é negativa à esquerda e positiva à direita de $p_{m}$, decorre que $p_{m}$ 
é ponto de minimo local de $\mathbf{f}$, isto é, existe vizinhança $V$ de $p_{m}$ tal que $\mathbf{f}(x) \geq \mathbf{f}\left(p_{m}\right), \forall x \in V$.

Analisando a variação do sinal de $\mathbf{f}^{\prime \prime}$, em que $\mathbf{f}^{\prime \prime}(x)=6 a x+2 b$, temos:

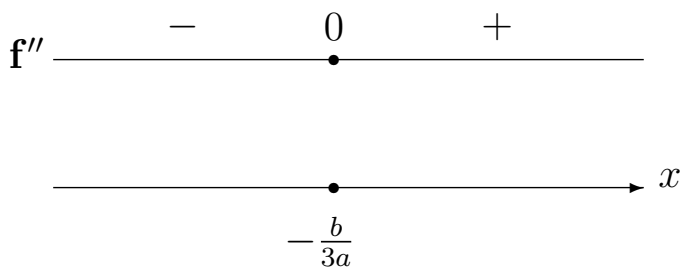

Como $\mathbf{f}^{\prime \prime}(x)<0$ para $\left.x \in\right]-\infty,-b / 3 a[$, segue que $\mathbf{f}$ tem concavidade para baixo em $]-\infty,-b / 3 a\left[\right.$; como $\mathbf{f}^{\prime \prime}(x)>0$ para $\left.x \in\right]-b / 3 a, \infty[$, segue que $\mathbf{f}$ tem concavidade para cima em $]-b / 3 a, \infty[$. O ponto $-b / 3 a$ é a abscissa de um ponto de inflexão (ponto da curva onde ocorre mudança de concavidade), já que $\mathbf{f}^{\prime \prime}(-b / 3 a)=6 a(-b / 3 a)+2 b=0$ e $\mathbf{f}^{\prime \prime \prime}(-b / 3 a)=6 a \neq 0$.

Portanto, a $1^{\mathrm{a}}$ forma do gráfico de $\mathbf{f}$ é:

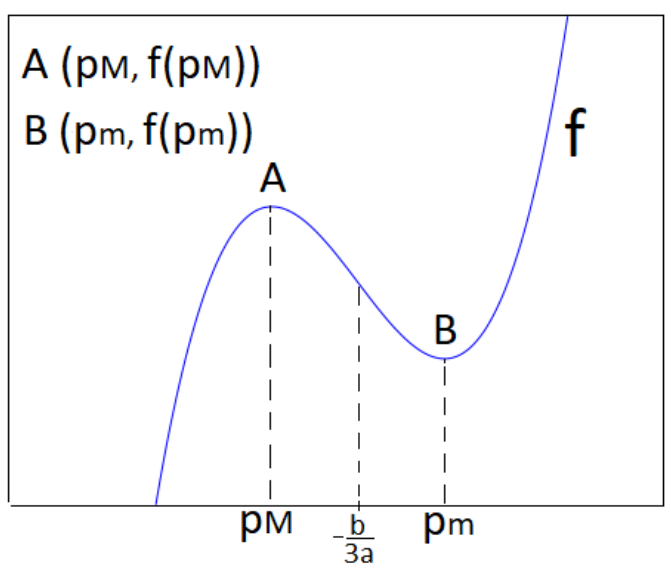

Figura 1: $1^{\mathrm{a}}$ forma do gráfico de $\mathrm{f}$

2. $2^{\mathrm{a}}$ forma $(\Delta=0)$

O ponto crítico é

$$
p=-\frac{b}{3 a} \text {. }
$$

Analisando a variação do sinal de $\mathbf{f}^{\prime}$, temos: 

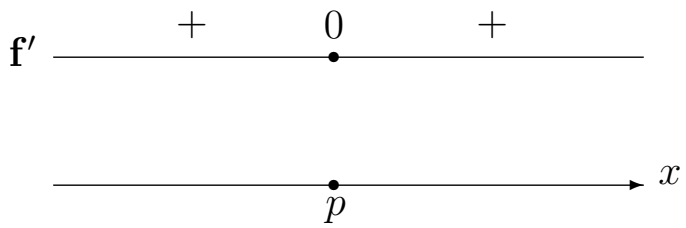

Como $\mathbf{f}^{\prime}(x)>0$ para $\left.x \in\right]-\infty, p[$ e para $x \in] p, \infty[$, segue que $\mathbf{f}$ é estritamente crescente nestes intervalos e que $p$ não é extremante (ponto de máximo local ou de mínimo local) de $\mathbf{f}$.

A variação do sinal de $\mathbf{f}^{\prime \prime}$ é a mesma da $1^{\mathrm{a}}$ forma.

Portanto, a $2^{\text {a }}$ forma do gráfico de $\mathbf{f}$ é:

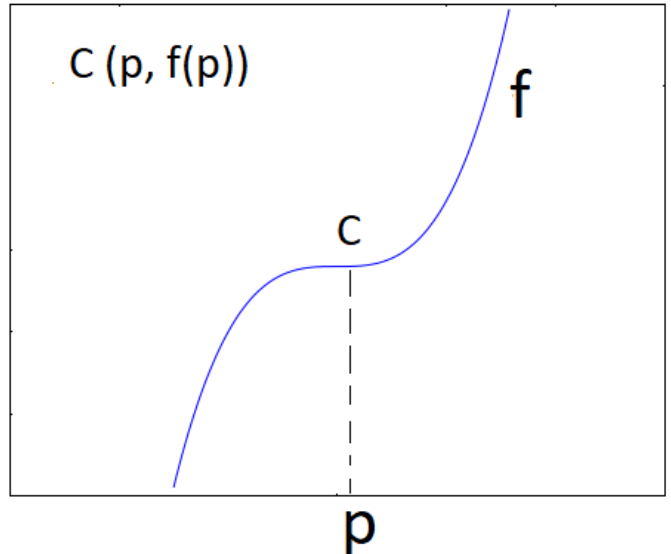

Figura 2: $2^{\mathrm{a}}$ forma do gráfico de $\mathrm{f}$

3. $3^{\text {a }}$ forma $(\Delta<0)$

Não existe ponto crítico.

Analisando a variação do sinal de $\mathbf{f}^{\prime}$, temos:

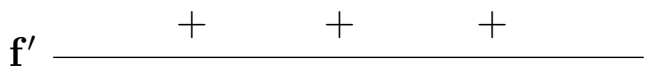

$x$

Como $\mathbf{f}^{\prime}(x)>0$ para $x \in \mathbb{R}$, segue que $\mathbf{f}$ é estritamente crescente em $\mathbb{R}$.

A variação do sinal de $\mathbf{f}^{\prime \prime}$ é a mesma da $1^{\text {a }}$ forma.

Portanto, a $3^{\text {a }}$ forma do gráfico de f é: 


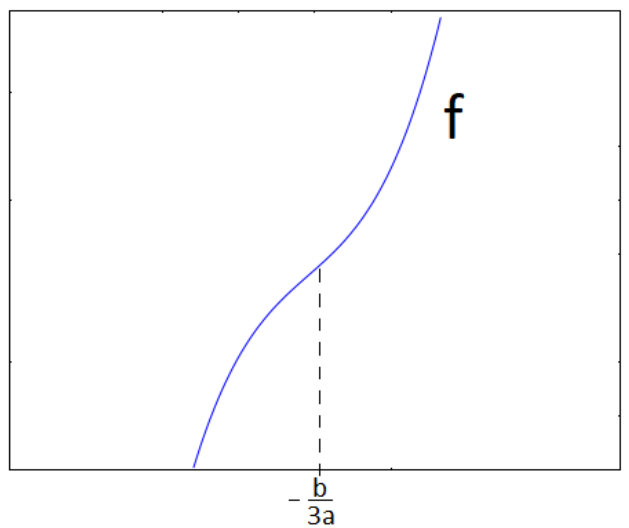

Figura 3: $3^{\text {a }}$ forma do gráfico de $\mathrm{f}$

Ao posicionar cada uma das 3 formas em relação ao eixo $x$, teremos as seguintes caracterizações (de 1. a 9.) das raízes, especificadas nas legendas das Figuras 4 a 12:

1. Os pontos A e B situam-se acima do eixo $x: \quad \mathbf{f}\left(p_{M}\right)>0$ e $\mathbf{f}\left(p_{m}\right)>0$

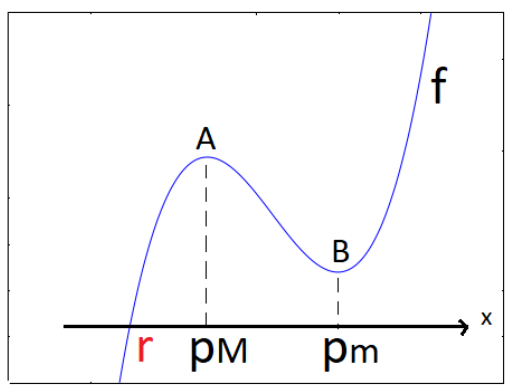

Figura 4: 1 raiz real $r\left(r<p_{M}\right)$ e 2 raízes complexas não reais conjugadas

2. O ponto A acima e o ponto B no eixo $x$ : $\quad \mathbf{f}\left(p_{M}\right)>0$ e $\quad \mathbf{f}\left(p_{m}\right)=0$

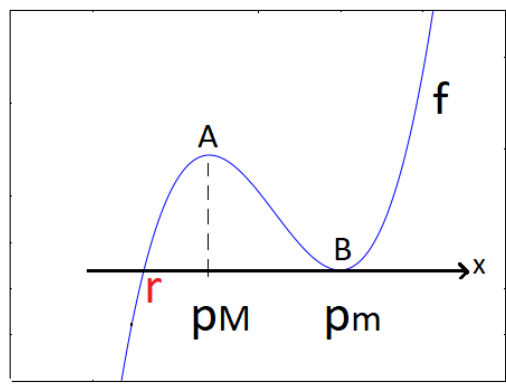

Figura 5: 1 raiz real $r\left(r<p_{M}\right)$ e $p_{m}$ é raiz real de multiplicidade 2 $p_{m}$ é raiz real de multiplicidade 2 , pois $\mathbf{f}$ não muda de sinal em $p_{m}$. 


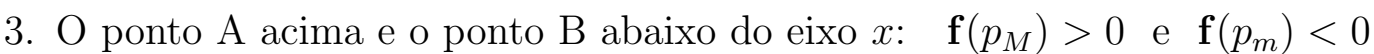

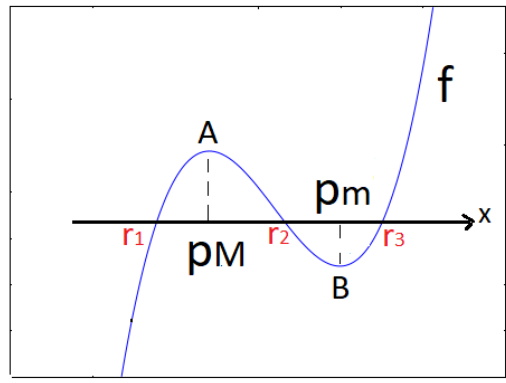

Figura 6: 3 raízes reais distintas: $r_{1}, r_{2}, r_{3}\left(r_{1}<p_{M}<r_{2}<p_{m}<r_{3}\right)$

4. O ponto A no e o ponto B abaixo do eixo $x$ : $\mathbf{f}\left(p_{M}\right)=0$ e $\mathbf{f}\left(p_{m}\right)<0$

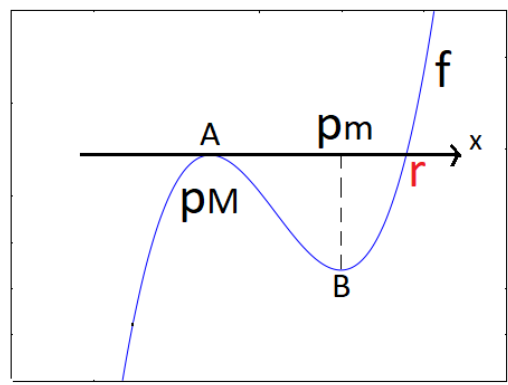

Figura 7: 1 raiz real $\mathrm{r}\left(r>p_{m}\right)$ e $p_{M}$ é raiz real de multiplicidade 2

$p_{M}$ é raiz real de multiplicidade 2 , pois $\mathbf{f}$ não muda de sinal em $p_{M}$.

5. Os pontos A e B situam-se abaixo do eixo $x$ : $\mathbf{f}\left(p_{M}\right)<0$ e $\mathbf{f}\left(p_{m}\right)<0$

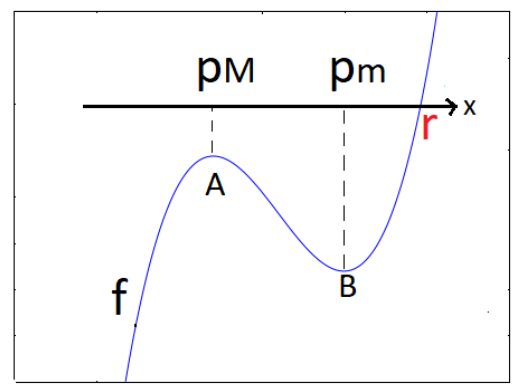

Figura 8: 1 raiz real $r\left(r>p_{m}\right)$ e 2 raízes complexas não reais conjugadas

6. O ponto C situa-se acima do eixo $x$ : $\mathbf{f}(p)>0$ 


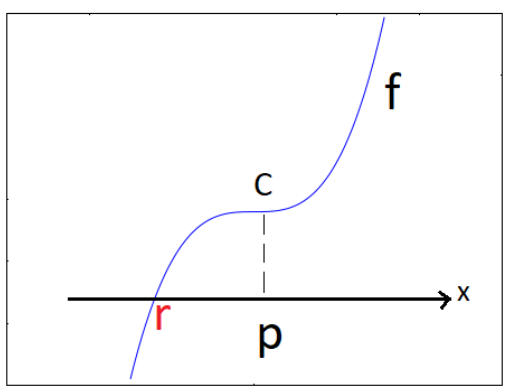

Figura 9: 1 raiz real $r(r<p)$ e 2 raízes complexas não reais conjugadas

7. O ponto C no eixo $x$ : $\mathbf{f}(p)=0$

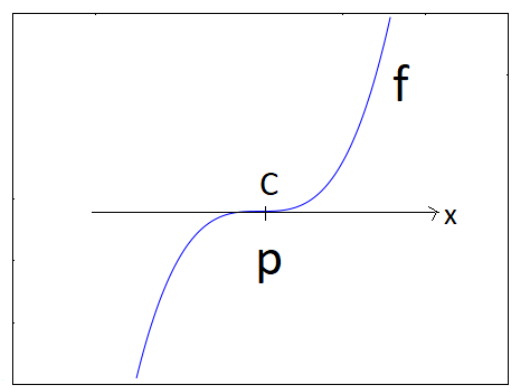

Figura 10: $p$ é raiz real de multiplicidade 3

8. O ponto C situa-se abaixo do eixo $x$ : $\mathbf{f}(p)<0$

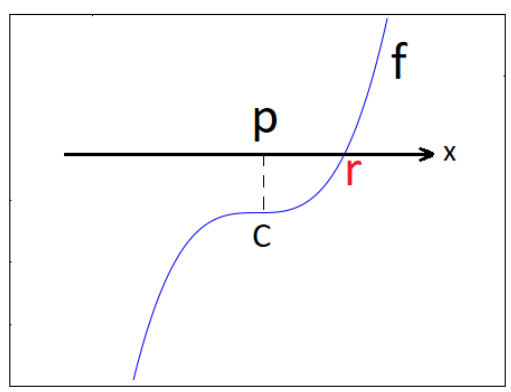

Figura 11: 1 raiz real $r(r>p)$ e 2 raízes complexas não reais conjugadas

9. Quando não existe ponto crítico 


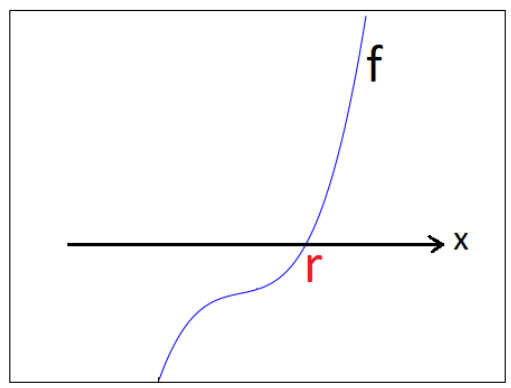

Figura 12: 1 raiz real $r$ e 2 raízes complexas não reais conjugadas

Procedimento para a caracterização das raízes de $f \in \mathbb{R}[x]$

- Determinar o(s) ponto(s) crítico(s)* de $\mathbf{f}: p$ ou $\left(p_{M}<p_{m}\right)$.

*Caso não exista(m), a caracterização é: 1 raiz real e 2 complexas conjugadas.

- Calcular: $\mathbf{f}(p)$ ou $\left(\mathbf{f}\left(p_{M}\right)\right.$ e $\left.\mathbf{f}\left(p_{m}\right)\right)$.

- Verificar as caracterizações: de 1. a 9. .

Exemplo 2.1. Caracterizar as raízes de $f \in \mathbb{R}[x]$ dado por $f(x)=x^{3}-4 x^{2}+x+6$.

Solução.

$$
\begin{gathered}
\mathbf{f}^{\prime}(x)=3 x^{2}-8 x+1=0 \\
p_{M}=\frac{4-\sqrt{13}}{3} \quad \text { e } \quad p_{m}=\frac{4+\sqrt{13}}{3} \\
\mathbf{f}\left(\frac{4-\sqrt{13}}{3}\right) \approx 7,93>0 \\
\mathbf{f}\left(\frac{4+\sqrt{13}}{3}\right) \approx-0,88<0
\end{gathered}
$$

Trata-se da caracterização 3.: 3 raízes reais: $r_{1}, r_{2}, r_{3}\left(r_{1}<p_{M}<r_{2}<p_{m}<r_{3}\right)$.

Exemplo 2.2. Caracterizar as raízes de $f \in \mathbb{R}[x]$ dado por $f(x)=x^{3}+6 x^{2}+12 x+$ 11.

Solução.

$$
\begin{gathered}
\mathbf{f}^{\prime}(x)=3 x^{2}+12 x+12=0 \\
p=-2 \\
\mathbf{f}(-2)=3>0
\end{gathered}
$$

Trata-se da caracterização 6.: 1 raiz real $r(r<-2)$ e 2 raízes complexas conjugadas. 
Exemplo 2.3. Caracterizar as raízes de $f \in \mathbb{R}[x]$ dado por $f(x)=x^{3}-5 x^{2}+7 x-3$.

Solução.

$$
\begin{gathered}
\mathbf{f}^{\prime}(x)=3 x^{2}-10 x+7=0 \\
p_{M}=1 \quad \text { e } \quad p_{m}=\frac{7}{3} \\
\mathbf{f}(1)=0 \\
\mathbf{f}\left(\frac{7}{3}\right) \approx-1,19<0
\end{gathered}
$$

Trata-se da caracterização 4.: Uma raiz real $r\left(r>\frac{7}{3}\right)$ e 1 é raiz real de multiplicidade 2 .

Exemplo 2.4. Caracterizar as raízes de $f \in \mathbb{R}[x]$ dado por $f(x)=x^{3}-6 x^{2}+12 x-8$.

Solução.

$$
\begin{gathered}
\mathbf{f}^{\prime}(x)=3 x^{2}-12 x+12=0 \\
p=2 \\
\mathbf{f}(2)=0
\end{gathered}
$$

Trata-se da caracterização $7 .: 2$ é raiz real de multiplicidade 3 .

Exemplo 2.5. Caracterizar as raizes de $f \in \mathbb{R}[x]$ dado por $f(x)=x^{3}+x^{2}+x-3$.

Solução.

$$
\begin{gathered}
\mathbf{f}^{\prime}(x)=3 x^{2}+2 x+1=0 \\
\Delta=4 .(1)^{2}-12.1 .1=-8<0
\end{gathered}
$$

Não existe ponto crítico. Trata-se da caracterização 9.: 1 raiz real $r$ e 2 raízes complexas conjugadas.

Observação 2.1. Quando $a<0$, temos $p_{m}$ (ponto de mínimo) $<p_{M}$ (ponto de máximo), e as 9 caracterizações das raízes de $f \in \mathbb{R}[x]$ estão especificadas abaixo: 
$\mathbf{f}\left(p_{m}\right) \mathbf{f}\left(p_{M}\right)$ Caracterização das raízes de $f \in \mathbb{R}[x]$

1. $>0>0$ 1 raiz real $r\left(r>p_{M}>p_{m}\right)$ e 2 raízes complexas conjugadas

2. $0>01$ raiz real $r\left(r>p_{M}>p_{m}\right)$ e $p_{m}$ é raiz real de multiplicidade 2

3. $<0>03$ raízes reais distintas: $r_{1}, r_{2}, r_{3}\left(r_{1}<p_{m}<r_{2}<p_{M}<r_{3}\right)$

4. $\quad<0 \quad 0 \quad 1$ raiz real $r\left(r<p_{m}<p_{M}\right)$ e $p_{M}$ é raiz real de multiplicidade 2

5. $<0<0 \quad 1$ raiz real $r\left(r<p_{m}<p_{M}\right)$ e 2 raízes complexas conjugadas

$\mathbf{f}(p) \quad$ Caracterização das raízes de $f \in \mathbb{R}[x]$

6. $\quad>0 \quad 1$ raiz real $r(r>p)$ e 2 raízes complexas conjugadas

7. $\quad 0 \quad$ p é raiz real de multiplicidade 3

8. $\quad<0 \quad 1$ raiz real $r(r<p)$ e 2 raízes complexas conjugadas

Caracterização das raízes de $f \in \mathbb{R}[x]$

9. $\quad \nexists\left(p, p_{m}, p_{M}\right) \quad 1$ raiz real $r$ e 2 raízes complexas conjugadas

\section{Alguns resultados}

Lema 3.1. Sejam $f \in \mathbb{R}[x]$ dado por $f(x)=a x^{3}+b x^{2}+c x, a>0,3 a c<b^{2}<4 a c$, $z_{1}$ e $\bar{z}_{1}$ as raizes complexas não reais conjugadas de $f\left(\operatorname{Re}\left(z_{1}\right)\right.$ e $\operatorname{Re}\left(\bar{z}_{1}\right)$ denotam a parte real de $z_{1}$ e de $\bar{z}_{1}$, respectivamente), $p_{m}$ o ponto de minimo local de $\mathbf{f}$ e $p_{M}$ o ponto de máximo local de $\mathbf{f}$. Temos:

i) $S e b<0$, então $\operatorname{Re}\left(z_{1}\right)=\operatorname{Re}\left(\bar{z}_{1}\right)>p_{m}>p_{M}>0$.

ii) $\operatorname{Se} b>0$, então $\operatorname{Re}\left(z_{1}\right)=\operatorname{Re}\left(\bar{z}_{1}\right)<p_{M}<p_{m}<0$.

Demonstração. De $a>0$ e $b^{2}<4 a c$ segue que as raízes complexas não reais conjugadas de $f(x)=a x^{3}+b x^{2}+c x=x\left(a x^{2}+b x+c\right)$ são

$$
z_{1}=-\frac{b}{2 a}+\frac{\sqrt{4 a c-b^{2}}}{2 a} i \quad, \quad \bar{z}_{1}=-\frac{b}{2 a}-\frac{\sqrt{4 a c-b^{2}}}{2 a} i, \quad i=\sqrt{-1}
$$

De $a>0$ e $b^{2}>3 a c$ segue que os pontos críticos de $\mathbf{f}$ são

$$
p_{1}=\frac{-2 b-\sqrt{4 b^{2}-12 a c}}{6 a} \text { e } p_{2}=\frac{-2 b+\sqrt{4 b^{2}-12 a c}}{6 a} \text {. }
$$

Como $\mathbf{f}^{\prime}\left(p_{1}\right)=0$ e $\mathbf{f}^{\prime \prime}\left(p_{1}\right)=6 a p_{1}+2 b=-\sqrt{4 b^{2}-12 a c}<0$ segue que $p_{1} \doteq p_{M}$ é o ponto de máximo local de $\mathbf{f}$. Como $\mathbf{f}^{\prime}\left(p_{2}\right)=0$ e $\mathbf{f}^{\prime \prime}\left(p_{2}\right)=6 a p_{2}+2 b=\sqrt{4 b^{2}-12 a c}>0$ segue que $p_{2} \doteq p_{m}$ é o ponto de mínimo local de $\mathbf{f}$. 
De $a>0$, obtemos

$$
p_{M}<p_{m}
$$

$a>0$ e $b^{2}<4 a c$ implicam $c>0$. Como $a c>0$ e $b^{2}>3 a c$, segue que

$$
0<4 b^{2}-12 a c<4 b^{2}
$$

i) Supondo $b<0$, de $(3)$ temos

$$
\begin{gathered}
\sqrt{4 b^{2}-12 a c}<-2 b \\
\Rightarrow-2 b-\sqrt{4 b^{2}-12 a c}>0 \\
\Rightarrow p_{M}>0 .
\end{gathered}
$$

Por fim, de $3 a c<b^{2}<4 a c, b<0$ e $a>0$ temos:

$$
\begin{aligned}
&\left\{\begin{array}{l}
b^{2}>3 a c \\
b^{2}<4 a c \\
b<0 \\
a>0
\end{array}\right. \Longrightarrow\left\{\begin{array}{l}
4 b^{2}-12 a c>0 \\
4 b^{2}-12 a c<b^{2} \\
b<0 \\
a>0
\end{array}\right. \\
& \Longrightarrow\left\{\begin{array}{l}
0<4 b^{2}-12 a c<b^{2} \\
b<0 \\
a>0
\end{array}\right. \\
& \Longrightarrow\left\{\begin{array}{l}
\sqrt{4 b^{2}-12 a c}<-b \\
a>0
\end{array}\right. \\
& \Longrightarrow-\frac{b-\sqrt{4 b^{2}-12 a c}>0}{6 a}+\frac{2 b-\sqrt{4 b^{2}-12 a c}}{6 a}>0 \\
& \Longrightarrow-\frac{b}{2 a}>\frac{-2 b+\sqrt{4 b^{2}-12 a c}}{6 a} \\
& \operatorname{Re}\left(z_{1}\right)>p_{m} \cdot
\end{aligned}
$$

Portanto, de (2), (4) e (5) segue a tese. 
ii) Supondo $b>0$, de (3) temos

$$
\begin{gathered}
\sqrt{4 b^{2}-12 a c}<2 b \\
\Rightarrow-2 b+\sqrt{4 b^{2}-12 a c}<0 \\
\Rightarrow p_{m}<0 .
\end{gathered}
$$

Por fim, de $3 a c<b^{2}<4 a c, b>0$ e $a>0$ temos:

$$
\begin{aligned}
\begin{cases}b^{2}>3 a c \\
b^{2}<4 a c \\
b>0 \\
a>0\end{cases} & \Longrightarrow\left\{\begin{array}{l}
4 b^{2}-12 a c>0 \\
4 b^{2}-12 a c<b^{2} \\
b>0 \\
a>0
\end{array}\right. \\
& \Longrightarrow\left\{\begin{array}{l}
0<4 b^{2}-12 a c<b^{2} \\
b>0 \\
a>0
\end{array}\right. \\
& \Longrightarrow\left\{\begin{array}{l}
\sqrt{4 b^{2}-12 a c}<b \\
a>0
\end{array}\right. \\
& \Longrightarrow \frac{-b+\sqrt{4 b^{2}-12 a c}<0}{6 a}-\frac{b}{2 a}+\frac{2 b+\sqrt{4 b^{2}-12 a c}}{6 a}<0 \\
& \Longrightarrow-\frac{b}{2 a}<\frac{-2 b-\sqrt{4 b^{2}-12 a c}}{6 a} \\
& \Longrightarrow \operatorname{Re}\left(z_{1}\right)<p_{M} \cdot
\end{aligned}
$$

Portanto, de (2), (6) e (7) segue a tese.

Teorema 3.1. Sejam $f \in \mathbb{R}[x]$ dado por $f(x)=a x^{3}+b x^{2}+c x, a>0$, $3 a c<b^{2}<$ $4 a c, z_{1} e \bar{z}_{1}$ as raízes complexas não reais conjugadas de $f, p_{m}$ o ponto de mínimo local de $\mathbf{f}$ e $p_{M}$ o ponto de máximo local de $\mathbf{f}$. Definindo $g=f+d, d \in \mathbb{R}$, temos:

i) $S e b<0$ e $d>0$, então as raízes complexas não reais conjugadas de $g$, $z_{2} e \bar{z}_{2}$, são tais que $\operatorname{Re}\left(z_{2}\right)=\operatorname{Re}\left(\bar{z}_{2}\right)>\operatorname{Re}\left(z_{1}\right)=\operatorname{Re}\left(\bar{z}_{1}\right)>p_{m}>p_{M}>0$, em que $p_{M}\left(p_{m}\right)$ é o ponto de máximo (mínimo) local da função polinomial $\mathbf{g}$ definida por $\mathrm{g}$.

ii) Se $b>0$ e $d<0$, então as raízes complexas não reais conjugadas de $g, z_{2}$ $e \bar{z}_{2}$, são tais que $\operatorname{Re}\left(z_{2}\right)=\operatorname{Re}\left(\bar{z}_{2}\right)<\operatorname{Re}\left(z_{1}\right)=\operatorname{Re}\left(\bar{z}_{1}\right)<p_{M}<p_{m}<0$, em que $p_{M}\left(p_{m}\right)$ é o ponto de máximo (mínimo) local da função polinomial $\mathbf{g}$ definida por $g$. Demonstração. 0 é raiz de $\mathbf{f}: \mathbb{R} \rightarrow \mathbb{R}$, pois $\mathbf{f}(0)=0$. E é a única raiz real. 
i) Da variação do sinal de $\mathbf{f}^{\prime}$ e de (2) e (4), podemos identificar os sinais de $\mathbf{f}$ :

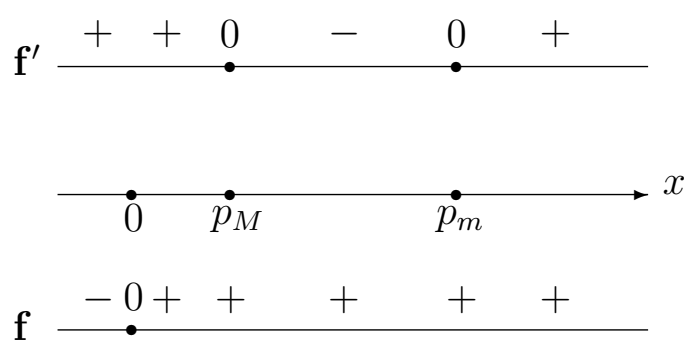

A Figura 13 corresponde ao gráfico de f. Dado $d>0$, deslocamos cada ponto do gráfico de f "d" unidades "para cima" para obter na Figura 14 o gráfico de g.

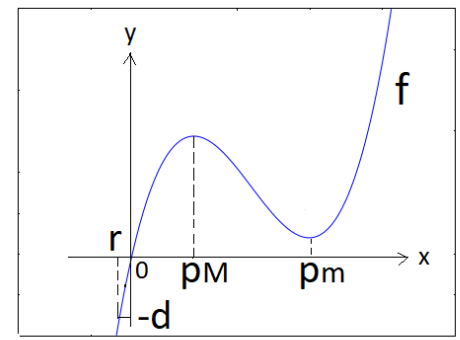

Figura 13: Gráfico de f

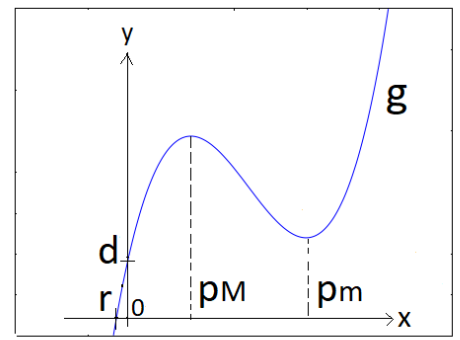

Figura 14: Gráfico de g

As raízes de $f$ são: $0, z_{1}=\alpha_{1}+\beta_{1} i, \bar{z}_{1}=\alpha_{1}-\beta_{1} i$, em que $\alpha_{1}>0, \beta_{1}>0$ (veja (1)). A soma das raízes de $f$ é

$$
2 \alpha_{1}=-\frac{b}{2 a}-\frac{b}{2 a}=-\frac{b}{a}
$$

Tome $-d \in \mathbb{R}$ (Figura 13). Como $\mathbf{f}(0)=0, \mathbf{f}$ é estritamente crescente em $]-\infty, 0[$ e é sobrejetora, segue que existe um único $r \in]-\infty, 0\left[\right.$ tal que $\mathbf{f}(r)=a r^{3}+b r^{2}+c r=$ $-d \Longrightarrow a r^{3}+b r^{2}+c r+d=0 \Longrightarrow \mathbf{g}(r)=0$. Isto é, $r$ é raiz real de $\mathbf{g}$ (Figura 14).

As raízes de $g$ são: $r, z_{2}=\alpha_{2}+\beta_{2} i, \bar{z}_{2}=\alpha_{2}-\beta_{2} i$, em que $\alpha_{2}, \beta_{2} \in \mathbb{R}, \beta_{2}>0$. Das Relações de Girard, tiramos que a soma das raízes de $g$ é

$$
r+2 \alpha_{2}=-\frac{b}{a}
$$

De (8) e (9) decorre que

$$
\alpha_{2}=\alpha_{1}-\frac{r}{2} \Longrightarrow \alpha_{2}>\alpha_{1} \text {, pois } r<0
$$

Os pontos de máximo local $\left(p_{M}\right)$ e de mínimo local $\left(p_{m}\right)$ de $\mathbf{f}$ são os mesmos de $\mathbf{g}$, pois $\mathbf{g}^{\prime}\left(p_{M}\right)=0, \mathbf{g}^{\prime \prime}\left(p_{M}\right)<0$ e $\mathbf{g}^{\prime}\left(p_{m}\right)=0, \mathbf{g}^{\prime \prime}\left(p_{m}\right)>0$. Como já dissemos, graficamente só se faz um deslocamento de "d" unidades "para cima" no gráfico de 
f para obter o de $\mathbf{g}$. Portanto, de (10) e do Lema 3.1 i) concluímos que

$$
\underbrace{\alpha_{2}}_{\operatorname{Re}\left(z_{2}\right)}>\underbrace{\alpha_{1}}_{\operatorname{Re}\left(z_{1}\right)}>p_{m}>p_{M}>0 .
$$

ii) Da variação do sinal de $\mathbf{f}^{\prime}$ e de (2) e (6), podemos identificar os sinais de $\mathbf{f}$ :

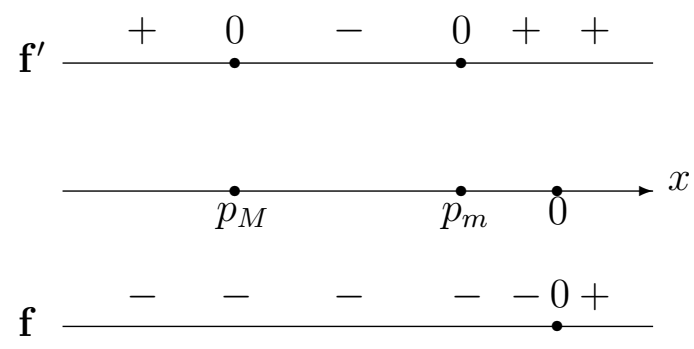

A Figura 15 corresponde ao gráfico de f. Dado $d<0$, deslocamos cada ponto do gráfico de f "d" unidades "para baixo" para obter na Figura 16 o gráfico de g.

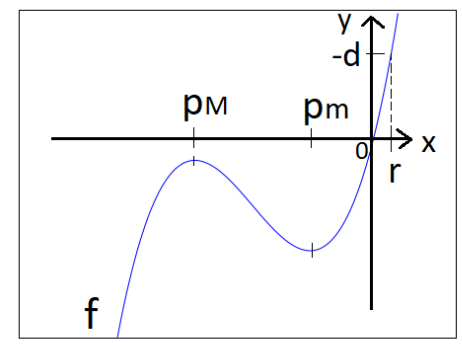

Figura 15: Gráfico de $\mathrm{f}$

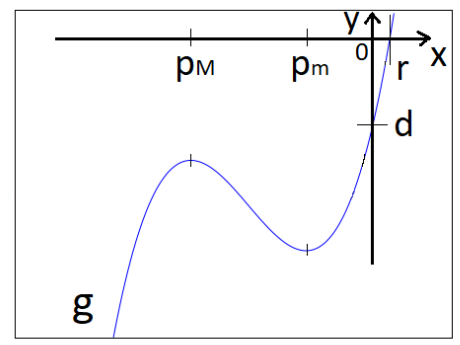

Figura 16: Gráfico de g

As raízes de $f$ são: $0, z_{1}=\alpha_{1}+\beta_{1} i, \bar{z}_{1}=\alpha_{1}-\beta_{1} i$, em que $\alpha_{1}<0, \beta_{1}>0$ (veja (1)). A soma das raízes de $f$ é

$$
2 \alpha_{1}=-\frac{b}{a}
$$

Tome $-d \in \mathbb{R}$ (Figura 15). Como $\mathbf{f}(0)=0$, f é estritamente crescente em $] 0, \infty[\mathrm{e}$ é sobrejetora, segue que existe um único $r \in] 0, \infty\left[\right.$ tal que $\mathbf{f}(r)=a r^{3}+b r^{2}+c r=-d$ $\Longrightarrow a r^{3}+b r^{2}+c r+d=0 \Longrightarrow \mathbf{g}(r)=0$. Isto é, $r$ é raiz real de $\mathbf{g}$ (Figura 16).

As raízes de $g$ são: $r, z_{2}=\alpha_{2}+\beta_{2} i, \bar{z}_{2}=\alpha_{2}-\beta_{2} i$, em que $\alpha_{2}, \beta_{2} \in \mathbb{R}, \beta_{2}>0$. Das Relações de Girard, tiramos que a soma das raízes de $g$ é

$$
r+2 \alpha_{2}=-\frac{b}{a}
$$


De (11) e (12) decorre que

$$
\alpha_{2}=\alpha_{1}-\frac{r}{2} \Longrightarrow \alpha_{2}<\alpha_{1} \text {, pois } r>0
$$

Os pontos de máximo local $\left(p_{M}\right)$ e de mínimo local $\left(p_{m}\right)$ de $\mathbf{f}$ são os mesmos de $\mathbf{g}$, pois $\mathbf{g}^{\prime}\left(p_{M}\right)=0, \mathbf{g}^{\prime \prime}\left(p_{M}\right)<0$ e $\mathbf{g}^{\prime}\left(p_{m}\right)=0, \mathbf{g}^{\prime \prime}\left(p_{m}\right)>0$. Como já dissemos, graficamente só se faz um deslocamento de "d" unidades "para baixo" no gráfico de f para obter o de g. Portanto, de (13) e do Lema 3.1 ii) concluímos que

$$
\underbrace{\alpha_{2}}_{\operatorname{Re}\left(z_{2}\right)}<\underbrace{\alpha_{1}}_{\operatorname{Re}\left(z_{1}\right)}<p_{M}<p_{m}<0 .
$$

Exemplo 3.1. A parte real das raízes complexas não reais conjugadas de $g(x)=$ $2 x^{3}-8,9 x^{2}+10 x+5$ é maior do que o ponto de mínimo local de $\mathbf{g}$.

\section{Solução.}

As condições do Teorema 3.1 i) estão satisfeitas, pois $a=2>0, b=-8,9<$ $0, c=10, d=5>0$ e $3.2 .10<(-8,9)^{2}<4.2 .10$.

Temos

$$
\begin{aligned}
& p_{M}=\frac{17,8-\sqrt{316,84-240}}{12} \approx 0,75 \text { (ponto de máximo local) } \\
& p_{m}=\frac{17,8+\sqrt{316,84-240}}{12} \approx 2,21 \text { (ponto de mínimo local). }
\end{aligned}
$$

Portanto, sendo $z_{2}$ raiz complexa não real de $g$, segue que

$$
\operatorname{Re}\left(z_{2}\right)>p_{m} \approx 2,21
$$

Com uma calculadora encontramos as raízes de $g\left(r \approx-0,37, z_{2} \approx 2,41+0,99 i\right.$, $\left.\bar{z}_{2} \approx 2,41-0,99 i\right)$. Veja a Figura 17 para o Exemplo 3.1 .

Exemplo 3.2. A parte real das raízes complexas não reais conjugadas de $g(x)=$ $2 x^{3}+8,9 x^{2}+10 x-5$ é menor do que o ponto de máximo local de $\mathbf{g}$.

\section{Solução.}

As condições do Teorema 3.1 ii) estão satisfeitas, pois $a=2>0, b=8,9>$ $0, c=10, d=-5<0$ e $3.2 .10<(8,9)^{2}<4.2 .10$. 
Temos

$$
\begin{aligned}
& p_{M}=\frac{-17,8-\sqrt{316,84-240}}{12} \approx-2,21 \quad \text { (ponto de máximo local), } \\
& p_{m}=\frac{-17,8+\sqrt{316,84-240}}{12} \approx-0,75 \quad \text { (ponto de mínimo local). }
\end{aligned}
$$

Portanto, sendo $z_{2}$ raiz complexa não real de $g$, segue que

$$
\operatorname{Re}\left(z_{2}\right)<p_{M} \approx-2,21
$$

Com uma calculadora encontramos as raízes de $g\left(r \approx 0,37, z_{2} \approx-2,41+0,99 i\right.$, $\left.\bar{z}_{2} \approx-2,41-0,99 i\right)$. Veja a Figura 18 para o Exemplo 3.2.

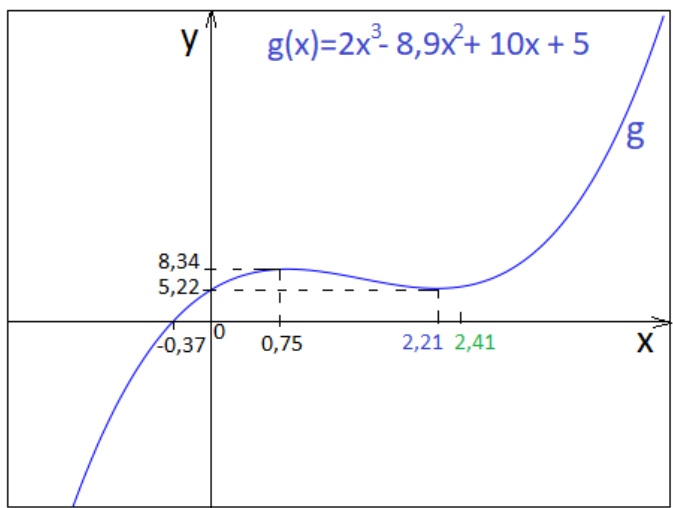

Figura 17: $\operatorname{Re}\left(z_{2}\right) \approx 2,41>p_{m} \approx 2,21$

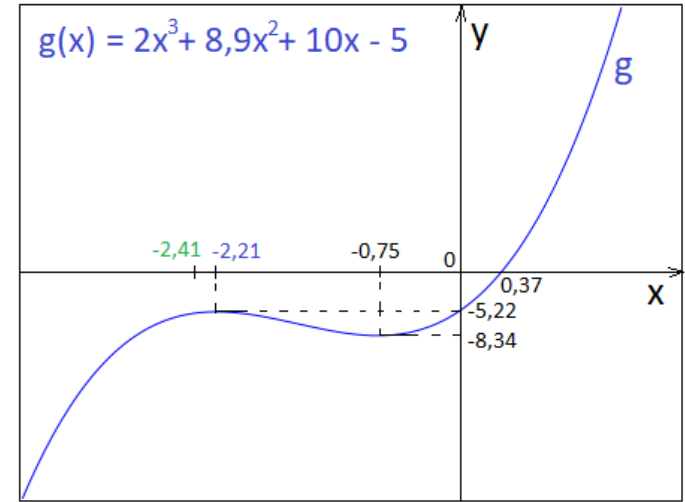

Figura 18: $\operatorname{Re}\left(z_{2}\right) \approx-2,41<p_{M} \approx-2,21$

Corolário 3.1. Sejam $f \in \mathbb{R}[x]$ dado por $f(x)=a x^{3}+b x^{2}+c x, a<0,3 a c<b^{2}<$ $4 a c, z_{1}$ e $\bar{z}_{1}$ as raízes complexas não reais conjugadas de $f, p_{m}$ o ponto de mínimo 
local de $\mathbf{f}$ e $p_{M}$ o ponto de máximo local de $\mathbf{f}$. Definindo $g=f+d, d \in \mathbb{R}$, temos:

i) $S e b>0$ e $d<0$, então as raízes complexas não reais conjugadas de $g, z_{2} e \bar{z}_{2}$, são tais que $\operatorname{Re}\left(z_{2}\right)=\operatorname{Re}\left(\bar{z}_{2}\right)>\operatorname{Re}\left(z_{1}\right)=\operatorname{Re}\left(\bar{z}_{1}\right)>p_{M}>p_{m}>0$, em que $p_{M}\left(p_{m}\right)$ é o ponto de máximo (mínimo) local da função polinomial $\mathbf{g}$ definida por $\mathrm{g}$.

ii) Se $b<0$ e $d>0$, então as raízes complexas não reais conjugadas de $g, z_{2}$ $e \bar{z}_{2}$, são tais que $\operatorname{Re}\left(z_{2}\right)=\operatorname{Re}\left(\bar{z}_{2}\right)<\operatorname{Re}\left(z_{1}\right)=\operatorname{Re}\left(\bar{z}_{1}\right)<p_{m}<p_{M}<0$, em que $p_{M}\left(p_{m}\right)$ é o ponto de máximo (mínimo) local da função polinomial $\mathbf{g}$ definida por $\mathrm{g}$.

Demonstração. i) Os coeficientes de $g$ do Corolário $3.1 i$ ) são opostos aos coeficientes de $g$ do Teorema $3.1 i$ ), que são $a>0, b<0, c>0$ e $d>0$. Logo, as raízes são as mesmas e o gráfico da função $\mathbf{g}$ do Corolário 3.1 i) é uma reflexão em relação ao eixo $x$ do gráfico da função $\mathbf{g}$ do Teorema 3.1 i). O ponto de máximo local da função g do Teorema 3.1 ) é o ponto de mínimo local da função g do Corolário 3.1 i); o ponto de mínimo local da função g do Teorema $3.1 i$ ) é o ponto de máximo local da função g do Corolário $3.1 \mathrm{i}$ ). Idem para $f$ : os coeficientes de $f$ do Corolário $3.1 i$ ) são opostos aos coeficientes de $f$ do Teorema $3.1 i$ ), logo suas raízes são as mesmas. Portanto, $\operatorname{Re}\left(z_{2}\right)=\operatorname{Re}\left(\bar{z}_{2}\right)>\operatorname{Re}\left(z_{1}\right)=\operatorname{Re}\left(\bar{z}_{1}\right)>p_{M}>p_{m}>0$. Veja a Figura 19.

ii) Faz-se a mesma análise para $g$ do Corolário 3.1 ii) e para $g$ do Teorema 3.1 ii), que têm sinais contrários. Idem para $f$.

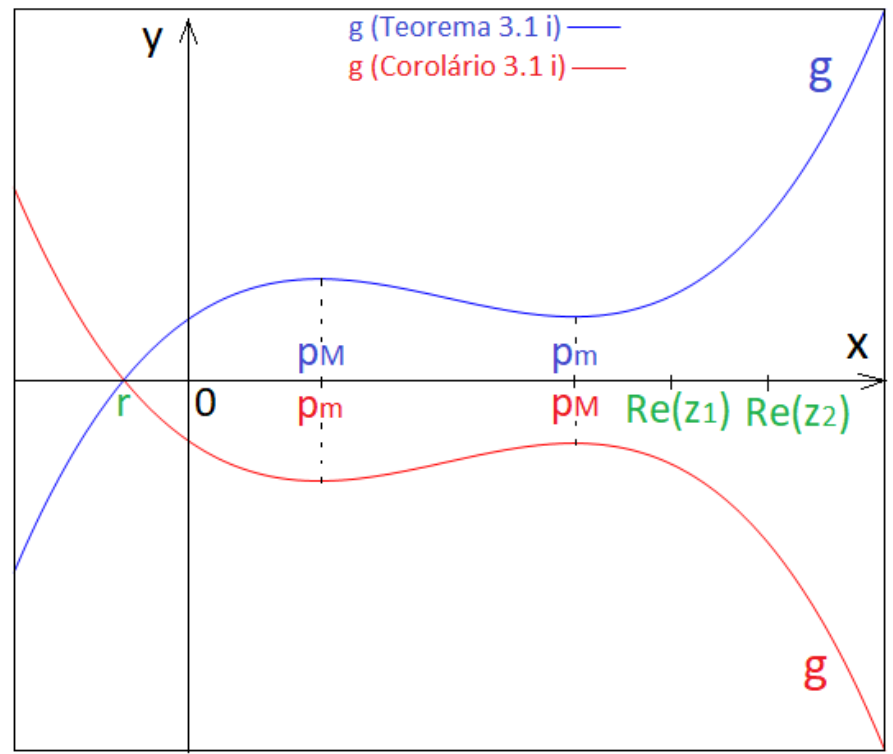

Figura 19: Comparativo entre o Teorema 3.1 i) e o Corolário 3.1 i) 
Lema 3.2. Sejam $f \in \mathbb{R}[x]$ dado por $f(x)=a x^{3}+b x^{2}+c x, a>0, b^{2}=3 a c, z_{1} e$ $\bar{z}_{1}$ as raízes complexas não reais conjugadas de $f$ e $p$ a abscissa do ponto de inflexão de f. Temos:

i) Se $b<0$, então $\operatorname{Re}\left(z_{1}\right)=\operatorname{Re}\left(\bar{z}_{1}\right)>p>0$.

ii) Se $b>0$, então $\operatorname{Re}\left(z_{1}\right)=\operatorname{Re}\left(\bar{z}_{1}\right)<p<0$.

Demonstração. De $a>0$ e $b^{2}=3 a c$ vem:

$$
z_{1}=-\frac{b}{2 a}+\frac{\sqrt{a c}}{2 a} i \quad, \quad \bar{z}_{1}=-\frac{b}{2 a}-\frac{\sqrt{a c}}{2 a} i
$$

e que o ponto crítico de $\mathbf{f}$ é

$$
p_{1}=-\frac{b}{3 a}
$$

Como $\mathbf{f}^{\prime \prime}\left(p_{1}\right)=6 a p_{1}+2 b=0$ e $\mathbf{f}^{\prime \prime \prime}\left(p_{1}\right)=6 a>0$, segue que $p_{1} \doteq p$ é a abscissa do ponto de inflexão de $\mathbf{f}$.

$$
\begin{aligned}
& \text { i) }\left\{\begin{array}{l}
a>0 \\
b<0
\end{array} \Longrightarrow-\frac{b}{2 a}>-\frac{b}{3 a}>0 \Longrightarrow \operatorname{Re}\left(z_{1}\right)>p>0 .\right. \\
& \text { ii) }\left\{\begin{array}{l}
a>0 \\
b>0
\end{array} \Longrightarrow-\frac{b}{2 a}<-\frac{b}{3 a}<0 \Longrightarrow \operatorname{Re}\left(z_{1}\right)<p<0 .\right.
\end{aligned}
$$

Teorema 3.2. Sejam $f \in \mathbb{R}[x]$ dado por $f(x)=a x^{3}+b x^{2}+c x, a>0, b^{2}=3 a c$, $z_{1}$ e $\bar{z}_{1}$ as raízes complexas não reais conjugadas de $f$ e $p$ a abscissa do ponto de inflexão de f. Definindo $g=f+d, d \in \mathbb{R}$, temos:

i) Se $b<0$ e $d>0$, então as raízes complexas não reais conjugadas de $g, z_{2} e$ $\bar{z}_{2}$, são tais que $\operatorname{Re}\left(z_{2}\right)=\operatorname{Re}\left(\bar{z}_{2}\right)>\operatorname{Re}\left(z_{1}\right)=\operatorname{Re}\left(\bar{z}_{1}\right)>p>0$, em que $p$ é a abscissa do ponto de inflexão da função polinomial $\mathbf{g}$ definida por $g$.

ii) $S e b>0$ e $d<0$, então as raízes complexas não reais conjugadas de $g, z_{2} e$ $\bar{z}_{2}$, são tais que $\operatorname{Re}\left(z_{2}\right)=\operatorname{Re}\left(\bar{z}_{2}\right)<\operatorname{Re}\left(z_{1}\right)=\operatorname{Re}\left(\bar{z}_{1}\right)<p<0$, em que p é a abscissa do ponto de inflexão da função polinomial $\mathbf{g}$ definida por $g$.

Demonstração. É similar à do Teorema 3.1, usando o Lema 3.2.

Exemplo 3.3. A parte real das raízes complexas não reais conjugadas de $g(x)=$ $x^{3}-3 x^{2}+3 x+4$ é maior do que a abscissa do ponto de inflexão de $\mathbf{g}$. 
Solução. As condições do Teorema $3.2 i$ ) estão satisfeitas, pois $a=1>0, b=$ $-3<0, c=3, d=4>0$ e $(-3)^{2}=3.1 .3$.

Temos

$$
p=1 \quad \text { (abscissa do ponto de inflexão). }
$$

Portanto, sendo $z_{2}$ raiz complexa não real de $g$, segue que

$$
\operatorname{Re}\left(z_{2}\right)>1
$$

Com uma calculadora encontramos as raízes de $g\left(r \approx-0,71, z_{2} \approx 1,85+1,48 i\right.$, $\left.\bar{z}_{2} \approx 1,85-1,48 i\right)$. Veja a Figura 20 para o Exemplo 3.3 .

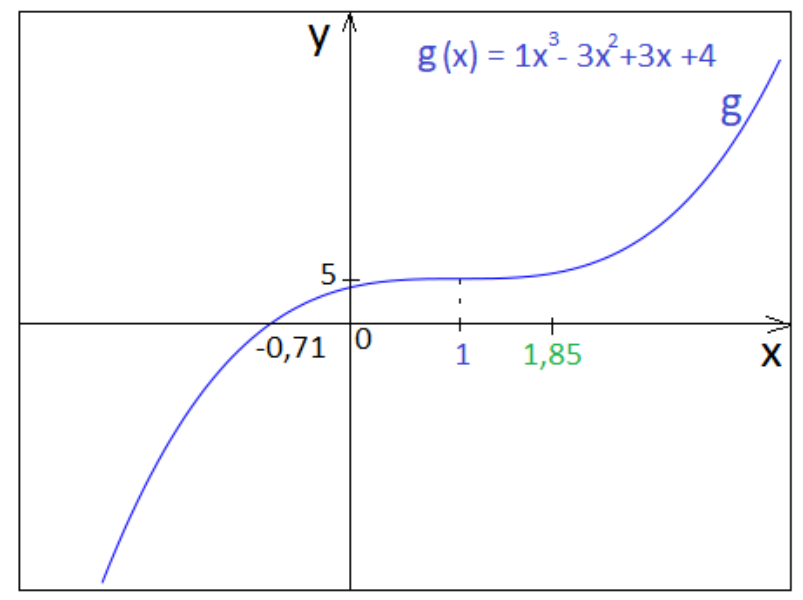

Figura 20: $\operatorname{Re}\left(z_{2}\right) \approx 1,85>p=1$

Corolário 3.2. Sejam $f \in \mathbb{R}[x]$ dado por $f(x)=a x^{3}+b x^{2}+c x, a<0, b^{2}=3 a c$, $z_{1}$ e $\bar{z}_{1}$ as raízes complexas não reais conjugadas de $f$ e $p$ a abscissa do ponto de inflexão de $\mathbf{f}$. Definindo $g=f+d, d \in \mathbb{R}$, temos:

i) $S e b>0$ e $d<0$, então as raízes complexas não reais conjugadas de $g, z_{2}$ e $\bar{z}_{2}$, são tais que $\operatorname{Re}\left(z_{2}\right)=\operatorname{Re}\left(\bar{z}_{2}\right)>\operatorname{Re}\left(z_{1}\right)=\operatorname{Re}\left(\bar{z}_{1}\right)>p>0$, em que p é a abscissa do ponto de inflexão da função polinomial $\mathbf{g}$ definida por $\mathrm{g}$.

ii) $S e b<0$ e $d>0$, então as raízes complexas não reais conjugadas de $g, z_{2} e$ $\bar{z}_{2}$, são tais que $\operatorname{Re}\left(z_{2}\right)=\operatorname{Re}\left(\bar{z}_{2}\right)<\operatorname{Re}\left(z_{1}\right)=\operatorname{Re}\left(\bar{z}_{1}\right)<p<0$, em que p é a abscissa do ponto de inflexão da função polinomial $\mathbf{g}$ definida por $g$.

Demonstração. É similar à do Corolário 3.1. 
Exemplo 3.4. A parte real das raízes complexas não reais conjugadas de $g(x)=$ $-x^{3}+3 x^{2}-3 x-4$ é maior do que a abscissa do ponto de inflexão de $\mathbf{g}$.

Solução. As condições do Corolário $3.2 i$ ) estão satisfeitas, pois $a=-1<0, b=$ $3>0, c=-3, d=-4<0$ e $3^{2}=3 .(-1) \cdot(-3)$.

Temos

$$
p=1 \quad \text { (abscissa do ponto de inflexão). }
$$

Portanto, sendo $z_{2}$ raiz complexa não real de $g$, segue que

$$
\operatorname{Re}\left(z_{2}\right)>1
$$

Com uma calculadora encontramos as raízes de $g\left(r \approx-0,71, z_{2} \approx 1,85+1,48 i\right.$, $\left.\bar{z}_{2} \approx 1,85-1,48 i\right)$. Veja a Figura 21 para o Exemplo 3.4 .

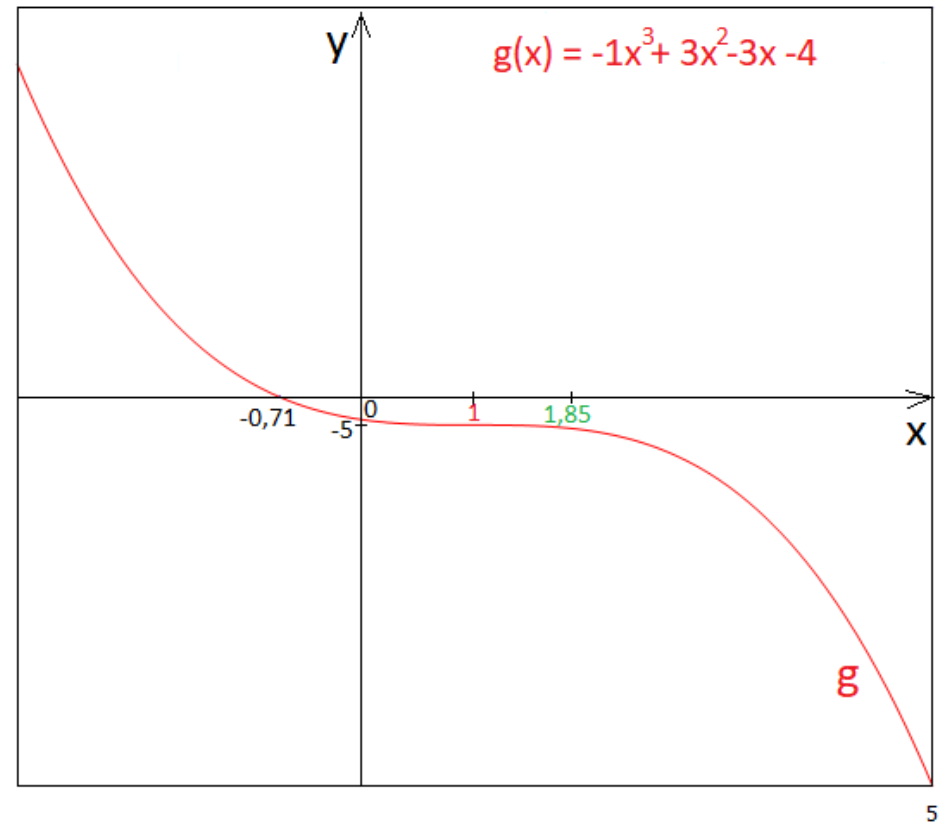

Figura 21: $\operatorname{Re}\left(z_{2}\right) \approx 1,85>p=1$

Todos os desenhos dos gráficos das funções neste artigo foram obtidos pelo software Maxima.

\section{Conclusão}

Na Seção 2 apresentamos um procedimento para caracterizar as raízes do polinômio do $3^{0}$ grau $f \in \mathbb{R}[x]$ calculando a função polinomial do $3^{0}$ grau $\mathbf{f}: \mathbb{R} \rightarrow \mathbb{R}$ no(s) 
seu(s) ponto(s) crítico(s), quando este(s) existe(m). Quando há pelo menos uma raiz real e pelo menos um ponto crítico podemos estabelecer uma ordem entre a raiz (as raízes) e o(s) ponto(s) crítico(s). Esta ordem está especificada em: i) nas legendas das Figuras 4 a 11, para $a>0$; ii) nas caracterizações 1. a 8. da Observação 2.1, para $a<0$. Na Seção 3 fizemos um comparativo entre a parte real das raízes complexas não reais conjugadas do polinômio do $3^{0}$ grau $g \in \mathbb{R}[x]$ e o(s) ponto(s) crítico(s) da função polinomial do $3^{0}$ grau $\mathbf{g}: \mathbb{R} \rightarrow \mathbb{R}$ para alguns casos particulares.

\section{Agradecimentos}

Agradeço ao parecerista anônimo pelas sugestões e pela correção que contribuíram para a melhoria do trabalho.

\section{Referências}

[1] GUIDORIZZI, H. L.: Um Curso de Cálculo, v.1, 6 $6^{\mathrm{a}}$ ed., São Paulo: LTC, 2001.

[2] IEZZI, G.: Fundamentos de Matemática Elementar, v.7, $4^{\mathrm{a}}$ ed., São Paulo: LTC, 1993.

[3] IEZZI, G.: Fundamentos de Matemática Elementar, v.6, $6^{\mathrm{a}}$ ed., São Paulo: LTC, 1995.

[4] IEZZI, G.; MURAKAMI, C.: Fundamentos de Matemática Elementar, v.1, $7^{\text {a }}$ ed., São Paulo: LTC, 1996.

[5] IEZZI, G.; MURAKAMI, C.; MACHADO, N. J.: Fundamentos de Matemática Elementar, v.8, 4${ }^{\mathrm{a}}$ ed., São Paulo: LTC, 1985.

[6] LIMA, E. L: A Equação do Terceiro Grau; Matemática Universitária, nํㅜㄴ, p.9-23, 1987.

[7] SOUZA, F.N. B: Uma abordagem geométrica para as equações cúbicas; Dissertação de Mestrado, Departamento de Matemática, Universidade Federal Rural de Pernambuco, Recife, 2013. 